Bull. Austral. Math. Soc.

VOL. 45 (1992) [9-23]

\title{
CONVOLUTION PROPERTIES OF A CLASS OF BOUNDED ANALYTIC FUNCTIONS
}

\author{
Zou Zhongzhu and Shigeyoshi OWA
}

Let $A$ be the class of functions $f(z)$ which are analytic in the unit disk $U$ with $f(0)=f^{\prime}(0)-1=0$. A subclass $\mathbf{S}(\lambda, M)(\lambda \geqslant 0, M>0)$ of $\mathbf{A}$ is introduced. The object of the present paper is to prove some interesting convolution properties of functions $f(z)$ belonging to the class $S(\lambda, M)$. Also a certain integral operator $J$ for $f(z)$ in the class $A$ is considered.

\section{INTRODUCTION AND LEMMAS}

Let $\mathbf{A}$ denote the class of analytic functions of the form

$$
f(z)=z+\sum_{n=2}^{\infty} a_{n} z^{n}
$$

in the unit disk $U=\{z:|z|<1\}$. We denote by $\mathbf{S}^{*}(\rho)$ and $\mathbf{K}(\rho)$ the subclasses of $\mathbf{A}$ whose members are starlike and convex of order $\rho(0 \leqslant \rho<1)$.

For a function $f(z) \in \mathbf{A}$, we say that $f(z)$ is in the class $\mathbf{S}(\lambda, M)$ if and only if it satisfies the condition

$$
\left|f^{\prime}(z)+\lambda z f^{\prime \prime}(z)-1\right|<M \quad(z \in \mathrm{U})
$$

for some $\lambda(\lambda \geqslant 0)$ and $M(M>0)$.

In the present paper, we prove some convolution properties of functions $f(z)$ belonging to the class $\mathbf{S}(\lambda, M)$. Some inclusion relations between $\mathbf{S}(\lambda, M)$ and other subclasses of $\mathbf{A}$ are obtained. We also obtain some new sufficient conditions for $f(z) \in \mathbf{S}^{*}(\rho)$. Finally, we discuss a class of certain integral operators on $\mathbf{A}$.

We need the following lemmas to derive our results.

Received 4 January 1991

The first author of the paper would like to thank Professors Wang Shugui, Jian Huaiyu, Li Guanghua and Xie Zhenghui for their helpful suggestions.

Copyright Clearance Centre, Inc. Serial-fee code: $0004-9729 / 92 \quad \$ A 2.00+0.00$. 
LEMMA 1. Let $\lambda \geqslant 0$ and $M>0$. If $p(z)$ is analytic in $\mathrm{U}$ with $p(0)=1$ and satisfies

$$
\left|p(z)+\lambda z p^{\prime}(z)-1\right|<M \quad(z \in \mathrm{U})
$$

then we have

$$
\begin{gathered}
|p(z)-1|<\frac{M}{1+\lambda} \quad(z \in \mathrm{U}), \\
\left|\frac{1}{z} \int_{0}^{z} p(t) d t-1\right|<\frac{M}{2(1+\lambda)} \quad(z \in \mathrm{U}),
\end{gathered}
$$

and

$$
\left|\frac{1}{z} \int_{0}^{z} p(t) d t-p(z)\right|<\frac{(3+2 \lambda) M}{2(1+\lambda)(1+2 \lambda)} \quad(z \in \mathrm{U}) .
$$

Inequalities in (2) and (3) cannot be improved.

ProOF: Let us define the function $p(z)$ by

$$
p(z)=1+\frac{M}{1+\lambda} w(z)
$$

where $w(z)$ is analytic in $U$ with $w(0)=0$. We wish to show that $|w(z)|<1$ for all $z \in \mathrm{U}$. If this is not true, then there exists a point $z_{0} \in \mathbf{U}$ satisfying

$$
\max _{|z| \leqslant\left|z_{0}\right|}|w(z)|=\left|w\left(z_{0}\right)\right|=1
$$

Then, by Jack's Lemma [1], we can write

$$
z_{0} w^{\prime}\left(z_{0}\right)=k w\left(z_{0}\right)
$$

where $k$ is real and $k \geqslant 1$. It follows that

and

$$
z_{0} p^{\prime}\left(z_{0}\right)=\frac{k M}{1+\lambda} w\left(z_{0}\right)
$$

$$
\left|p\left(z_{0}\right)+\lambda z_{0} p^{\prime}\left(z_{0}\right)-1\right|=\frac{1+\lambda k}{1+\lambda} M \geqslant M \text {. }
$$

This contradicts the condition (1), and hence we conclude that $|w(z)|<1$ for all $z \in U$. Therefore, by using (5), we know that (2) holds true.

In view of Schwarz' Lemma and (2), we have

$$
|p(z)-1| \leqslant \frac{M}{1+\lambda}|z| \quad(z \in \mathbf{U})
$$


and hence

$$
\left|\int_{0}^{z} p(t) d t-z\right|=\left|\int_{0}^{z}(p(t)-1) d t\right| \leqslant \int_{0}^{|z|} \frac{M}{1+\lambda} t d t=\frac{M}{2(1+\lambda)}|z|^{2} .
$$

This implies that

$$
\left|\frac{1}{z} \int_{0}^{z} p(t) d t-1\right|<\frac{M}{2(1+\lambda)} \quad(z \in \mathbf{U})
$$

Further, let

$$
p(z)-\frac{1}{z} \int_{0}^{z} p(t) d t=\frac{(3+2 \lambda) M}{2(1+\lambda)(1+2 \lambda)} w(z)
$$

where $w(z)$ is analytic in $U$ with $w(0)=0$. We can prove that $|w(z)|<1$ for all $z \in \mathrm{U}$. In fact, if this is not true, then using the same way as in the above there exists a point $z_{0} \in \mathbf{U}\left(z_{0} \neq 0\right)$ such that $\left|w\left(z_{0}\right)\right|=1$ and $z_{0} w^{\prime}\left(z_{0}\right)=k w\left(z_{0}\right)$, where $k \geqslant 1$. From (1) and (6), we obtain

$$
\begin{aligned}
\mid p\left(z_{0}\right) & +\lambda z_{0} p^{\prime}\left(z_{0}\right)-1 \mid \\
= & \left|\frac{1}{z_{0}} \int_{0}^{z_{0}} p(t) d t+\frac{(3+2 \lambda) M w\left(z_{0}\right)}{2(1+\lambda)(1+2 \lambda)}+\frac{(3+2 \lambda) \lambda(k+1)}{2(1+\lambda)(1+2 \lambda)} M w\left(z_{0}\right)-1\right| \\
< & =
\end{aligned}
$$

that is,

$$
\left|\frac{1}{z_{0}} \int_{0}^{z_{0}} p(t) d t-1+\frac{3+2 \lambda}{2(1+\lambda)(1+2 \lambda)}(1+\lambda+k \lambda) M w\left(z_{0}\right)\right|<M .
$$

Hence, we have

$$
\left|\frac{1}{z_{0}} \int_{0}^{z_{0}} p(t) d t-1\right|>\frac{3+2 \lambda}{2(1+\lambda)} M-M=\frac{M}{2(1+\lambda)} .
$$

This contradicts (3) and hence $|w(z)|<1$ for all $z \in \mathrm{U}$. This follows (4) with (6).

Since the function $p_{0}(z)=1+(M /(1+\lambda)) z$ satisfies the condition (1), we see that the inequalities in (2) and (3) cannot be improved. Thus we complete the proof of Lemma 1.

Let $A_{n}=\left(a_{i j}\right)_{n n}$ denote the real symmetrical matrix of order $n$. Jian Huaiyu has showed that $\left|A_{n}\right| \geqslant 0$, if $A_{n}$ satisfies the conditions:
(i) $a_{i j} \geqslant a_{i j+1} \geqslant 0$
$(i=1,2,3, \ldots, n ; i \leqslant j \leqslant n-1)$,
(ii) $a_{i+1} i+1 \geqslant a_{i i}$
$(i=1,2,3, \ldots, n-1)$,
(iii) $a_{i j} \geqslant a_{i-1} j$
$\left(i=1,2,3, \ldots, n_{i} i \leqslant j \leqslant n\right)$, 
and

(iv) $a_{i j}-a_{i j+1} \geqslant a_{i-1 j}-a_{i-1 j+1} \quad(i=1,2,3, \ldots, n ; i \leqslant j \leqslant n-1)$.

In fact, the case $a_{11}=0$ is trivial. If $a_{11}>0$, then we have

$$
\left|A_{n}\right|=\left|\begin{array}{cccc}
a_{11} & a_{12} & \ldots & a_{1 n} \\
0 & a_{22}^{\prime} & \ldots & a_{2 n}^{\prime} \\
\ldots \ldots & \ldots & \ldots & \ldots \\
0 & a_{n 2}^{\prime} & \ldots & a_{n n}^{\prime}
\end{array}\right|=a_{11}\left|\begin{array}{ccc}
a_{22}^{\prime} & \ldots & a_{2 n}^{\prime} \\
\ldots \ldots & \ldots & \ldots \\
a_{n 2}^{\prime} & \ldots & a_{n n}^{\prime}
\end{array}\right|
$$

where

$$
a_{i j}^{\prime}=a_{i j}-\frac{a_{i 1}}{a_{11}} a_{1 j} \quad(i, j=1,2,3, \ldots, n) .
$$

By the hypothesis, we see that

$$
\left(\begin{array}{ccc}
a_{22}^{\prime} & \ldots & a_{2 n}^{\prime} \\
\ldots \ldots & \ldots & \ldots \\
a_{n 2}^{\prime} & \ldots & a_{n n}^{\prime}
\end{array}\right)
$$

is a real symmetrical matrix of order $n-1$ and satisfies the conditions (i) - (iv). Hence we can prove that $\left|A_{n}\right| \geqslant 0$ by mathematical induction.

LEMMA 2. Let $b_{0}>0, b_{n} \geqslant 0$, and $b_{n-1}-b_{n} \geqslant b_{n}-b_{n+1} \geqslant 0, n=1,2,3, \ldots$ If

$$
p(z)=\frac{b_{0}}{2}+\sum_{n=1}^{\infty} b_{n} z^{n}
$$

then $\operatorname{Re}(p(z))>0(z \in \mathrm{U})$.

Proof: We can write

$$
p(z)=\frac{b_{0}}{2}\left\{1+\sum_{n=1}^{\infty} c_{n} z^{n}\right\}
$$

with $c_{n}=2 b_{n} / b_{0}(n=1,2,3, \ldots)$. Adopting the convention that $c_{0}=2, c_{-n}=c_{n}$ $(n \geqslant 1)$, we have that

$$
A_{m+1}=\left(\begin{array}{ccccc}
c_{0} & c_{1} & c_{2} & \ldots & c_{m} \\
c_{1} & c_{0} & c_{1} & \ldots & c_{m-1} \\
\ldots \ldots & \ldots & \ldots & \ldots \ldots \ldots \ldots \ldots \\
c_{i} & c_{i-1} & c_{i-2} & \ldots & c_{i-m} \\
\ldots \ldots \ldots \ldots \ldots \ldots \ldots \ldots \ldots \ldots & \ldots \ldots \\
c_{m} & c_{m-1} & c_{m-2} & \ldots & c_{0}
\end{array}\right) \quad(i=0,1,2, \ldots, m)
$$


is a real symmetrical matrix of order $m+1$, and satisfies the conditions (i) - (iv). Hence we can prove that $A_{m+1}$ is a semi-positive definite matrix by the mathematical induction.

Since, for $m=1,2,3, \ldots$ and $\lambda_{k} \in \mathrm{C}(0 \leqslant k \leqslant m)$, we have

$$
\begin{gathered}
R_{m}=\sum_{k=0}^{m} \sum_{q=0}^{m} C_{k-q} \lambda_{k} \bar{\lambda}_{q}=\lambda^{\prime} A_{m+1} \lambda, \\
\lambda=\left(\begin{array}{c}
\bar{\lambda}_{0} \\
\bar{\lambda}_{1} \\
\vdots \\
\bar{\lambda}_{m}
\end{array}\right)
\end{gathered}
$$

and hence $R_{m} \geqslant 0$; this implies that

$$
\operatorname{Re}\left\{1+\sum_{n=1}^{\infty} c_{n} z^{n}\right\}>0 \quad(z \in U)
$$

so Lemma 2 is completed.

EXAMPLE. If $\lambda \geqslant 0$ and

$$
f(z)=z+\sum_{n=2}^{\infty} \frac{1}{1-\lambda+n \lambda} z^{n}
$$

then

$$
\operatorname{Re}\left\{\frac{f(z)}{z}\right\}>\frac{4 \lambda^{2}+3 \lambda+1}{2(1+\lambda)(1+2 \lambda)} \quad(z \in \mathrm{U}) .
$$

Proof: Let $b_{0}=(1+3 \lambda) /((1+\lambda)(1+2 \lambda))$ and $b_{n}=1 /(1+n \lambda), n=$ $1,2,3, \ldots$ Clearly, the sequence $\left\{b_{n}\right\}_{0}^{\infty}$ satisfies the conditions in Lemma 2 , and hence

$$
\operatorname{Re}\left\{\frac{1+3 \lambda}{2(1+\lambda)(1+2 \lambda)}+\sum_{n=1}^{\infty} \frac{1}{1+n \lambda} z^{n}\right\}>0 \quad(z \in U) .
$$

The conclusion follows from (8) at once.

\section{The Class $\mathbf{S}(\lambda, M)$}

Let a function $f(z)$ be in the class $S(\lambda, M)$. Setting $p(z)=f^{\prime}(z)$ in Lemma 1, by (2) and (3), we obtain

$$
\left|f^{\prime}(z)-1\right|<\frac{M}{1+\lambda} \quad(z \in \mathrm{U})
$$

and

$$
\left|\frac{f(z)}{z}-1\right|<\frac{M}{2(1+\lambda)} \quad(z \in \mathbf{U})
$$


respectively. From (10), we see that $\mathbf{S}(\lambda, M)$ is a class of bounded analytic functions in $\mathrm{U}$. If $M \leqslant 1+\lambda$, by (9), $\mathbf{S}(\lambda, M) \subset \mathbf{C}$, the usual class of close-to-convex functions in $U$. From (9), we also obtain

Proposition 1. Let $0 \leqslant \lambda_{2} \leqslant \lambda_{1}$ and $\lambda_{1}>0$. Then

$$
\mathbf{S}\left(\lambda_{1}, M\right) \subset \mathbf{S}\left(\lambda_{2}, M\right) \text {. }
$$

Theorem 2. Let $f(z) \in \mathbf{S}(\lambda, M)$ and $g(z) \in \mathbf{A}$ with $\operatorname{Re}\{g(z) / z\}>1 / 2$ $(z \in \mathrm{U})$; then $h(z)=(f * g)(z) \in \mathbf{S}(\lambda, M)$, where $(f * g)(z)$ denotes the convolution (or Hadamard product) of functions $f(z)$ and $g(z)$.

Proof: According to Herglotz Theorem, we have

$$
\frac{g(z)}{z}=\int_{T} \frac{1}{1-z \tau} d \mu(\tau),
$$

where $\mu$ is a probability measure on the unit circle $T$. Since

$$
h^{\prime}(z)+\lambda z h^{\prime \prime}(z)-1=\left(f^{\prime}(z)+\lambda z f^{\prime \prime}(z)-1\right) * \frac{g(z)}{z},
$$

we obtain

$$
h^{\prime}(z)+\lambda z h^{\prime \prime}(z)-1=\int_{T}\left(f^{\prime}(\tau z)+\lambda \tau z f^{\prime \prime}(\tau z)-1\right) d \mu(\tau) .
$$

Moreover, we have

$$
\left|h^{\prime}(z)+\lambda z h^{\prime \prime}(z)-1\right|<\int_{T} M d \mu(\tau)=M
$$

which shows $h(z) \in \mathbf{S}(\lambda, M)$.

Corollary 1. Let $f(z) \in \mathbf{S}(\lambda, M), g(z) \in \mathbf{S}(\lambda, M)$ and $M \leqslant 1+\lambda$. Then $h(z)=(f * g)(z) \in \mathbf{S}(\lambda, M)$, that is, $\mathbf{S}(\lambda, M)$ is closed for the convolution (or Hadamard product) when $M \leqslant 1+\lambda$.

ProOF: By means of (10), we have $\operatorname{Re}\{f(z) / z\}>1 / 2(z \in \mathrm{U})$, and hence the conclusion immediately follows from Theorem 2.

Next, we derive

Theorem 3. Let $f(z) \in \mathbf{S}(\lambda, M), g(z) \in \mathbf{S}(\lambda, M)$, and $h(z)=(f * g)(z)$.

(i) If $M \leqslant 1+\lambda$, then $h(z) \in \mathbf{S}^{*}(0)$ and satisfies

$$
\left|\frac{z h^{\prime}(z)}{h(z)}-1\right|<1 \quad(z \in \mathrm{U}) .
$$

(ii) If either $\lambda \geqslant 1 / 3$ with $M \leqslant(1+\lambda) / \sqrt{2}$, or $0<\lambda \leqslant 1 / 3$ with $M \leqslant$ $\sqrt{2 \lambda(1+\lambda)}$, then $h(z) \in \mathrm{K}(0)$. 
Proof: Defining the functions $f(z)$ and $g(z)$ by $f(z)=z+\sum_{n=2}^{\infty} a_{n} z^{n}$ and $g(z)=$ $z+\sum_{n=2}^{\infty} b_{n} z^{n}$, respectively, we have

$$
h(z)=(f * g)(z)=z+\sum_{n=2}^{\infty} a_{n} b_{n} z^{n} .
$$

(i) From (9), we obtain that

$$
\iint_{\mathrm{U}}\left|f^{\prime}(z)-1\right|^{2} d x d y=\pi \sum_{n=2}^{\infty} n\left|a_{n}\right|^{2}<\pi\left(\frac{M}{1+\lambda}\right)^{2} .
$$

Hence

$$
\sum_{n=2}^{\infty} n\left|a_{n}\right|^{2}<1
$$

Similarly, we have

$$
\sum_{n=2}^{\infty} n\left|b_{n}\right|^{2}<1
$$

By means of the Cauchy-Schwarz inequality, we obtain

$$
\sum_{n=2}^{\infty} n\left|a_{n} b_{n}\right| \leqslant\left(\sum_{n=2}^{\infty} n\left|a_{n}\right|^{2}\right)^{1 / 2}\left(\sum_{n=2}^{\infty} n\left|b_{n}\right|^{2}\right)^{1 / 2}<1 .
$$

Therefore, we know that $h(z) \in \mathrm{S}^{*}(0)$, so $h(z) / z \neq 0(z \in \mathrm{U})$. It follows from (14) that

or

$$
\sum_{n=2}^{\infty} n\left|a_{n} b_{n}\right||z|^{n-1}<1 \quad(z \in \mathrm{U})
$$

$$
\sum_{n=2}^{\infty}(n-1)\left|a_{n} b_{n}\right||z|^{n-1}<1-\sum_{n=2}^{\infty}\left|a_{n} b_{n}\right||z|^{n-1} \quad(z \in U) .
$$

This implies that

$$
\left|\sum_{n=2}^{\infty}(n-1) a_{n} b_{n} z^{n-1}\right|<\left|1+\sum_{n=2}^{\infty} a_{n} b_{n} z^{n-1}\right| \quad(z \in U),
$$


that is, that

$$
\left|h^{\prime}(z)-\frac{h(z)}{z}\right|<\left|\frac{h(z)}{z}\right| \quad(z \in \mathrm{U}) .
$$

Consequently, we obtain that

$$
\left|\frac{z h^{\prime}(z)}{h(z)}-1\right|<1 \quad(z \in U) .
$$

(ii) Since $f(z) \in \mathrm{S}(\lambda, M)$, we have

$$
\left|\sum_{n=2}^{\infty} n(1-\lambda+n \lambda) a_{n} z^{n-1}\right|<M \quad(z \in \mathrm{U}),
$$

and hence

$$
\begin{gathered}
\iint_{\mathrm{U}}\left|\sum_{n=2}^{\infty} n(1-\lambda+n \lambda) a_{n} z^{n-1}\right|^{2} d x d y=\pi \sum_{n=2}^{\infty} n(1-\lambda+n \lambda)^{2}\left|a_{n}\right|^{2} \\
<\pi M^{2}
\end{gathered}
$$

Since $\lambda \geqslant 1 / 3$ with $M \leqslant(1+\lambda) / \sqrt{2}$, or $0<\lambda \leqslant 1 / 3$ with $M \leqslant \sqrt{2 \lambda(1+\lambda)}$, we can prove that $(1-\lambda+n \lambda)^{2} \geqslant n M^{2}$ for every $n \geqslant 2$, and hence by (15) we have

$$
\sum_{n=2}^{\infty} n^{2}\left|a_{n}\right|^{2}<1
$$

Similarly

$$
\sum_{n=2}^{\infty} n^{2}\left|b_{n}\right|^{2}<1
$$

Therefore, we see that

$$
\sum_{n=2}^{\infty} n^{2}\left|a_{n} b_{n}\right| \leqslant\left(\sum_{n=2}^{\infty} n^{2}\left|a_{n}\right|^{2}\right)^{1 / 2}\left(\sum_{n=2}^{\infty} n^{2}\left|b_{n}\right|^{2}\right)^{1 / 2}<1 .
$$

This implies that $h(z)$ belongs to the class $\mathrm{K}(0)$.

From the proof of (i) in Theorem 3, we have

Corollary 2. If

$$
F(z)=z+\sum_{n=2}^{\infty} c_{n} z^{n}
$$


is in the class $\mathbf{A}$ with

then

$$
\sum_{n=2}^{\infty} n\left|c_{n}\right| \leqslant 1
$$

$$
\left|\frac{z F^{\prime}(z)}{F(z)}-1\right|<1 \quad(z \in \mathrm{U})
$$

Letting $\lambda=M-1=0$ in (i) of Theorem 3, we have

Corollary 3. Let $f(z) \in \mathbf{A}$ and $g(z) \in \mathbf{A}$ with $\left|f^{\prime}(z)-1\right|<1 \quad(z \in \mathrm{U})$ and $\left|g^{\prime}(z)-1\right|<1 \quad(z \in \mathbf{U})$. Then $h(z)=(f * g)(z) \in \mathbf{S}^{*}(0)$ and

$$
\left|\frac{z h^{\prime}(z)}{h(z)}-1\right|<1 \quad(z \in \mathrm{U})
$$

THEOREM 4. Let $f(z) \in \mathbf{S}(\lambda, M)$.

(i) If $M \leqslant 2(1+\lambda) / \sqrt{5}$, then $f(z) \in \mathbf{S}^{*}(0)$.

(ii) If $M \leqslant(1+2 \lambda) / 2$, then $\left|z f^{\prime}(z) / f(z)-1\right|<1 \quad(z \in \mathrm{U})$.

(iii) If $M \leqslant 2(1+\lambda)(1+2 \lambda) /(5+6 \lambda)$, then $f(z) \in \mathbf{S}^{*}(1 / 2)$.

Proof: (i) Since $M /(1+\lambda) \leqslant 2 / \sqrt{5}<1$, in view of $(9)$, we obtain $\operatorname{Re}\left\{f^{\prime}(z)\right\}>$ $0(z \in \mathrm{U})$, and

$$
\left|\arg f^{\prime}(z)\right|<\sin ^{-1}\left(\frac{M}{1+\lambda}\right) \leqslant \sin ^{-1}\left(\frac{2}{\sqrt{5}}\right)<\frac{\pi}{2} \quad(z \in \mathrm{U}) .
$$

By (10), we have $\operatorname{Re}\{f(z) / z\}>0(z \in U)$, and

$$
\left|\arg \frac{f(z)}{z}\right|<\sin ^{-1}\left(\frac{1}{\sqrt{5}}\right)<\frac{\pi}{2} \quad(z \in \mathbf{U}) .
$$

Noting that

$$
\sin \left(\sin ^{-1}\left(\frac{2}{\sqrt{5}}\right)+\sin ^{-1}\left(\frac{1}{\sqrt{5}}\right)\right)=1
$$

we have

$$
\left|\arg \frac{z f^{\prime}(z)}{f(z)}\right| \leqslant\left|\arg f^{\prime}(z)\right|+\left|\arg \frac{f(z)}{z}\right|<\frac{\pi}{2} \quad(z \in \mathrm{U}),
$$

which implies $f(z) \in \mathrm{S}^{*}(0)$.

(ii) Setting $p(z)=f^{\prime}(z)$ in Lemma 1 , we have by (4)

$$
\left|f^{\prime}(z)-\frac{f(z)}{z}\right|<\frac{3+2 \lambda}{4(1+\lambda)} \quad(z \in U) .
$$


Since (10) gives

$$
\left|\frac{f(z)}{z}\right|>1-\frac{1+2 \lambda}{4(1+\lambda)}=\frac{3+2 \lambda}{4(1+\lambda)} \quad(z \in U)
$$

we have

$$
\left|f^{\prime}(z)-\frac{f(z)}{z}\right|<\left|\frac{f(z)}{z}\right| \quad(z \in \mathbf{U})
$$

which proves

$$
\left|\frac{z f^{\prime}(z)}{f(z)}-1\right|<1 \quad(z \in \mathbf{U})
$$

(iii) It is sufficient to prove that

$$
\left|\frac{z f^{\prime}(z)}{f(z)}-1\right|<\left|\frac{z f^{\prime}(z)}{f(z)}\right| \quad(z \in \mathbf{U}) .
$$

Since (10) leads to

$$
\left|\frac{f(z)}{z}\right|>\frac{4(1+\lambda)}{5+6 \lambda} \quad(z \in \mathrm{U})
$$

we see that $f(z) / z \neq 0(z \in U)$. Therefore, we only need to show that

$$
\left|f^{\prime}(z)-\frac{f(z)}{z}\right|<\left|f^{\prime}(z)\right| \quad(z \in \mathbf{U}) .
$$

With the aid of (4) and (9), we obtain

$$
\left|f^{\prime}(z)-\frac{f(z)}{z}\right|<\frac{3+2 \lambda}{5+6 \lambda} \quad(z \in \mathrm{U})
$$

and

$$
\left|f^{\prime}(z)\right|>\frac{3+2 \lambda}{5+6 \lambda} \quad(z \in \mathrm{U})
$$

Thus we prove the inequality (16).

REMARK. Taking $\lambda=0$ and $M=1$ in (i) of Theorem 4, we obtain Theorem 2 and Theorem 3 by Mocanu [2]. Further, letting $M=1$ in (ii) of Theorem 4, we obtain the main result by Mocanu [2], that is, Theorem 4.

Making $\lambda=0$ in (iii) of Theorem 4, we have

Corollary 4. $S(0,2 / 5) \subset S^{*}(1 / 2)$.

By Corollary 4, we see 
Corollary 5. $S(1,2 / 5) \subset \mathbf{K}(1 / 2)$.

Next, in view of (9), we derive

ThEOREM 5. If $z f^{\prime}(z) \in \mathbf{S}(\lambda, M)$, then $f(z) \in \mathbf{S}(1, M /(1+\lambda))$. Conversely, if $f(z) \in \mathbf{S}(\lambda, M)$, then $z f^{\prime}(z) \in \mathbf{S}(0,2 M / \lambda(1+\lambda))$ when $0<\lambda \leqslant 1$, and $z f^{\prime}(z) \in$ $\mathrm{S}(0,2 M /(1+\lambda))$ when $\lambda \geqslant 1$.

THEOREM 6. Let

$$
f(z)=z+\sum_{k=2}^{\infty} a_{k} z^{k}
$$

belong to the class $\mathrm{S}(\lambda, M)$. Then, for every $n \geqslant 1$, the $n$th partial sum $f_{n}(z)$ of $f(z)$ satisfies

$$
\left|\frac{f_{n}(z)}{z}-1\right|<\frac{M}{1+\lambda} \quad(z \in \mathrm{U})
$$

and

$$
\left|f_{n}^{\prime}(z)-1\right|<M \quad(z \in \mathrm{U}),
$$

where $\lambda \geqslant 1$.

Proof: We define the function $g(z)$ by $g(z)=\log (1 /(1-z))$. Then, we have $g(z) \in \mathrm{K}(1 / 2)$, and $\operatorname{Re}\left\{g_{n}(z) / z\right\}>1 / 2(z \in \mathrm{U})$ by Singh [3, Theorem 2], where $g_{n}(z)$ denotes the $n$th partial sum of $g(z)$.

(i) Since $f(z) \in \mathbf{S}(\lambda, M)$, by (9) and the equality

$$
\frac{f_{n}(z)}{z}-1=\left(f^{\prime}(z)-1\right) * \frac{g_{n}(z)}{z} \quad(z \in \mathbf{U}),
$$

in the same method as Theorem 2, we obtain

$$
\left|\frac{f_{n}(z)}{z}-1\right|<\frac{M}{1+\lambda} \quad(z \in \mathrm{U}) .
$$

(ii) By Proposition 1, we see that $f(z) \in \mathbf{S}(1, M)$, and hence

$$
\left|1+\sum_{k=2}^{\infty} k^{2} a_{k} z^{k-1}-1\right|<M \quad(z \in \mathrm{U})
$$

Since

$$
f_{n}^{\prime}(z)-1=\left(1+\sum_{k=2}^{\infty} k^{2} a_{k} z^{k-1}-1\right) * \frac{g_{n}(z)}{z} \quad(z \in U),
$$

by the same way as the part (i), we obtain

$$
\left|f_{n}^{\prime}(z)-1\right|<M \quad(z \in \mathrm{U})
$$

for all $\lambda \geqslant 1$.

Corollary 6. If $f(z) \in \mathbf{S}(\lambda, 1)$, then $f_{n}(z) \in \mathbf{C}$ for all $\lambda \geqslant 1$ and for every $n \geqslant 1$. 


\section{INTEGRAL OPERATORS}

We now discuss integral operators

$$
g(z)=J(f)(z)=\frac{\gamma+1}{z^{\gamma}} \int_{0}^{z} t^{\gamma-1} f(t) d t \quad(\gamma>-1)
$$

for $f(z) \in A$. Writing $\gamma=1 / \lambda-1(\lambda>0)$, we see that

$$
f(z)=(1-\lambda) g(z)+\lambda z g^{\prime}(z)
$$

and

$$
f^{\prime}(z)=g^{\prime}(z)+\lambda z g^{\prime \prime}(z)
$$

Clearly, if $\lambda>0$ and $g(z) \in \mathbf{S}(\lambda, M)$, then we observe that $f(z)$ defined by (17) is in the class $\mathbf{S}(0, M)$. Conversely, we have

THEOREM 7. The integral operator $J$ defined by (17) satisfies

$$
J: \mathrm{S}(1 /(1+\gamma), M) \longrightarrow \mathrm{S}(1 /(1+\gamma),(1+\gamma) M /(2+\gamma))
$$

Proof: Setting $\lambda=1 /(1+\gamma)$ and $p(z)=g^{\prime}(z)+\lambda z g^{\prime \prime}(z)$, we see from (19) that

$$
f^{\prime}(z)+\lambda z f^{\prime \prime}(z)-1=p(z)+\lambda z p^{\prime}(z)-1
$$

Suppose that $f(z) \in \mathbf{S}(\lambda, M)=\mathbf{S}(1 /(1+\gamma), M)$. Then it follows from (2) that

$$
|p(z)-1|<\frac{M}{1+\lambda} \quad(z \in U)
$$

and hence $g(z) \in S(\lambda, M /(1+\lambda))$. This completes the proof of Theorem 7 .

THEOREM 8. Let $M \leqslant 1+\lambda,-1<\gamma=1 / \lambda-1 \leqslant 0$, and $\lambda \geqslant 1$. If $f(z) \epsilon$ $\mathbf{S}(\lambda, M)$ and $g(z)$ is defined by (17), then $(g * h)(z) \in \mathbf{K}(0)$ for every $h(z) \in \mathbf{S}(\lambda, M)$.

Proof: Defining the functions $g(z)$ and $h(z)$ by

$$
g(z)=z+\sum_{n=2}^{\infty} a_{n} z^{n} \text { and } h(z)=z+\sum_{n=2}^{\infty} b_{n} z^{n}
$$

(18) leads to

$$
f(z)=z+\sum_{n=2}^{\infty}(1-\lambda+n \lambda) a_{n} z^{n} \in \mathbf{S}(\lambda, M)
$$


Therefore, from (12), we have

$$
\sum_{n=2}^{\infty} n(1-\lambda+n \lambda)^{2}\left|a_{n}\right|^{2}<\left(\frac{M}{1+\lambda}\right)^{2}<1 .
$$

Noting that $\lambda \geqslant 1$, we have

$$
\sum_{n=2}^{\infty} n^{3}\left|a_{n}\right|^{2}<1
$$

Further, by (12) we obtain

$$
\sum_{n=2}^{\infty} n\left|b_{n}\right|^{2}<1
$$

Consequently, we know that

$$
\sum_{n=2}^{\infty} n^{2}\left|a_{n} b_{n}\right|<1
$$

which implies that $(g * h)(z) \in \mathbf{K}(0)$.

Next, we prove

TheOREM 9. If $f(z) \in \mathbf{A}$ satisfies $\operatorname{Re}\{f(z) / z\}>\rho(\rho<1 ; z \in \mathrm{U})$, then the function $g(z)$ defined by (17) satisfies

$$
\operatorname{Re}\left\{\frac{g(z)}{z}\right\}> \begin{cases}\frac{2+4 \rho+5 \rho \gamma+\rho \gamma^{2}}{(2+\gamma)(3+\gamma)} & (-1<\gamma \leqslant 0) \\ \frac{1+2 \rho+2 \rho \gamma}{3+2 \rho} & (\gamma>0),\end{cases}
$$

for $z \in U$.

Proof: Letting

$$
g(z)=z+\sum_{n=2}^{\infty} a_{n} z^{n}
$$

and $\gamma=1 / \lambda-1(\lambda>0),(18)$ gives

$$
\operatorname{Re}\left\{\frac{f(z)}{z}\right\}=\operatorname{Re}\left\{1+\sum_{n=2}^{\infty}(1-\lambda+n \lambda) a_{n} z^{n-1}\right\}>\rho \quad(z \in U)
$$

Hence we have

$$
\operatorname{Re}\left\{1+\frac{1}{2(1-\rho)} \sum_{n=2}^{\infty}(1-\lambda+n \lambda) a_{n} z^{n-1}\right\}>\frac{1}{2} \quad(z \in \mathrm{U})
$$


Note that

(21)

$$
\frac{g(z)}{z}=\left\{1+\frac{1}{2(1-\rho)} \sum_{n=2}^{\infty}(1-\lambda+n \lambda) a_{n} z^{n-1}\right\} *\left\{1+2(1-\rho) \sum_{n=2}^{\infty} \frac{z^{n-1}}{1-\lambda+n \lambda}\right\} .
$$

Thus (7) leads to

$$
\operatorname{Re}\left\{1+2(1-\rho) \sum_{n=2}^{\infty} \frac{z^{n-1}}{1-\lambda+n \lambda}\right\}>\frac{2 \lambda^{2}+(1-3 \lambda) \rho}{(1+\lambda)(1+2 \lambda)} \quad(z \in \mathrm{U}) .
$$

Combining (20), (21) and (22), in a similar way to Theorem 2, we obtain

$$
\operatorname{Re}\left\{\frac{g(z)}{z}\right\}>\frac{2 \lambda^{2}+(1+3 \lambda) \rho}{(1+\lambda)(1+2 \lambda)} \quad(z \in \mathrm{U})
$$

for all $\lambda>0$, that is,

$$
\operatorname{Re}\left\{\frac{g(z)}{z}\right\}>\frac{2+4 \rho+5 \rho \gamma+\rho \gamma^{2}}{(2+\gamma)(3+\gamma)} \quad(z \in \mathrm{U})
$$

for all $\gamma>-1$. But for $\gamma>0$, that is, for $0<\lambda<1$, we have

$$
\frac{2+4 \rho+5 \rho \gamma+\rho \gamma^{2}}{(2+\gamma)(3+\gamma)}<\frac{1+2 \rho+2 \rho \gamma}{3+2 \gamma} \text {. }
$$

Applying Jack's Lemma [1], we can prove

$$
\operatorname{Re}\left\{\frac{g(z)}{z}\right\}>\frac{1+2 \rho+2 \rho \gamma}{3+2 \gamma} \quad(z \in \mathbf{U})
$$

for $\gamma>0$.

REMARK. The above inequality (23) was recently proved by Owa and Nunokawa [4] when $0 \leqslant \rho<1$ and $\gamma>-1$.

With the help of the proof of Theorem 9, we have

Theorem 10. If $f(z) \in \mathbf{A}$ satisfies $\operatorname{Re}\left\{f^{\prime}(z)\right\}>\rho(\rho<1 ; z \in \mathrm{U})$, then the function $g(z)$ defined by (17) satisfies

$$
\operatorname{Re}\left\{g^{\prime}(z)\right\}> \begin{cases}\frac{2+4 \rho+5 \rho \gamma+\rho \gamma^{2}}{(2+\gamma)(3+\gamma)} & (-1<\gamma \leqslant 0) \\ \frac{1+2 \rho+2 \rho \gamma}{3+2 \gamma} & (\gamma>0)\end{cases}
$$

for $z \in \mathbf{U}$.

REMARK. The second inequality in Theorem 10 was proved by Owa and Nunokawa [4] when $0 \leqslant \rho<1$ and $\gamma>-1$. 


\section{REFERENCES}

[1] I.S. Jack, 'Functions starlike and convex of order $\alpha$ ', J. London Math. Soc. 3 (1971), 469-474.

[2] P.T. Mocanu, 'Some starlikeness conditions for analytic functions', Rev. Roumaine Math. Pures Appl. 33 (1988), 117-124.

[3] R. Singh, 'On the partial sums of convex functions of order 1/2', Proc. Amer. Math. Soc. 102 (1988), 541-545.

[4] S. Owa and M. Nunokawa, 'Properties of certain analytic functions', Math. Japon. 33 (1988), 577-582.

Department of Mathematics

Huaihua Teachers' College

Hunan 418008

People's Republic of China
Department of Mathematics

Kinki University

Higashi-Osaka, Osaks 577

Japan 